\title{
420-MHz Cr:forsterite femtosecond ring laser and continuum generation in the $1-2-\mu \mathrm{m}$ range
}

\author{
I. Thomann, A. Bartels, K. L. Corwin, N. R. Newbury, L. Hollberg, and Scott A. Diddams \\ National Institute of Standards and Technology, 325 Broadway, Boulder, Colorado 80305
}

J. W. Nicholson and M. F. Yan

OFS Laboratories, 600-700 Mountain Avenue, Murray Hill, New Jersey 07974

\begin{abstract}
We demonstrate a chromium-doped forsterite femtosecond ring laser that generates 30 -fs pulses at a $420-\mathrm{MHz}$ repetition rate with nearly $500 \mathrm{~mW}$ of average power. The compact solid-state design and broad spectral output make this laser attractive for telecommunications applications in the $1.3-1.5-\mu \mathrm{m}$ region. Additional spectral broadening of the laser output in highly nonlinear optical fiber leads to octave-spanning spectra ranging from 1.06 to $2.17 \mu \mathrm{m}$. The octave is reached at a level of $18 \mathrm{~dB}$ below the peak. The underlying optical frequency comb can be linked to existing optical frequency standards.
\end{abstract}

Chromium-doped forsterite is a promising broadband solid-state gain medium centered near $1.3-\mu \mathrm{m}$ wavelength. Not long after its introduction, Seas et $a l .^{1}$ first demonstrated Kerr lens mode locking in Cr:forsterite. Since that time progress in generating shorter pulses has been made by several groups. $^{2-4}$ While femtosecond Cr:forsterite lasers have proved to be useful tools in biological imaging, ${ }^{5,6}$ our interests are in the fields of optical frequency metrology and phase control of femtosecond lasers. ${ }^{7}$

With a few exceptions, nearly all optical frequency metrology that employs femtosecond lasers has been based on Ti:sapphire lasers used in combination with microstructure optical fibers. ${ }^{7}$ However, the $1.3-1.6-\mu \mathrm{m}$ regime is a natural direction in which to apply these new tools, because wavelength and frequency references in this regime are important for fiber-based communications. ${ }^{8}$ For optical frequency metrology it is desirable to have a broadband femtosecond laser with both a high repetition rate and high average power, as this increases the power per optical mode of the associated frequency-domain comb. With these factors in mind, we have developed a broadband Cr:forsterite femtosecond laser that employs dispersioncompensating mirrors in a $420-\mathrm{MHz}$ ring cavity. To our knowledge, this is the first ring configuration as well as the highest repetition rate yet reported for a femtosecond Cr:forsterite laser. The already broad spectrum emitted by the laser is further broadened in highly nonlinear optical fiber (HNLF), leading to an octave-spanning spectrum.

The design of our compact, prismless six-mirror ring cavity (Fig. 1) follows that of earlier work with Ti:sapphire. ${ }^{9}$ The repetition rate in the present configuration is $420 \mathrm{MHz}$, and we believe it could readily be increased to $\sim 1 \mathrm{GHz}$. The pump source is an ytterbium fiber laser operating at $1075 \mathrm{~nm}$ with an output power of $10 \mathrm{~W}$ focused to a waist with a radius of $\approx 15 \mu \mathrm{m}$ in the crystal. The 10 -mm-long
Cr:forsterite crystal (doping of $0.2 \%$ by weight, absorption coefficient $\alpha=1.1 \mathrm{~cm}^{1}$ at $1075 \mathrm{~nm}$, estimated figure of merit $\geq 120$ ) is placed at Brewster's angle between two concave mirrors with a 5 -cm radius of curvature. The crystal is oriented such that the pump beam polarization is along the $b$ axis $\left(P_{b n m}\right.$ notation). The crystal is mounted in a copper block that is cooled to $\sim 0{ }^{\circ} \mathrm{C}$ to achieve higher gain and purged with nitrogen to prevent water condensation. The entire laser is mounted on a temperaturecontrolled baseplate that is placed in a thick-walled box to isolate it from the laboratory environment. The pump threshold for $\mathrm{cw}$ operation is $\approx 1.5 \mathrm{~W}$, and the slope efficiency is $\approx 5.5 \%$. Mode-locked operation is initiated by tapping on one of the cavity mirrors, and, once it is started, unidirectional stable mode-locked operation is maintained for $4-8 \mathrm{~h}$.

The crystal introduces $+185-\mathrm{fs}^{2}$ group-delay dispersion (GDD) and $+620-\mathrm{fs}^{3}$ third-order dispersion at the center wavelength of $1280 \mathrm{~nm} .{ }^{10}$ Intracavity dispersion compensation is provided by a combination of chirped mirrors (GDD $=55 \pm 20 \mathrm{fs}^{2}$ from 1200 to $1415 \mathrm{~nm}$ ) and Gires-Tournois interferometer mirrors $\left(\mathrm{GDD}=280 \pm 10 \mathrm{fs}^{2}\right.$ from 1200 to $1325 \mathrm{~nm}$ ). By use of different mirror combinations, a range of

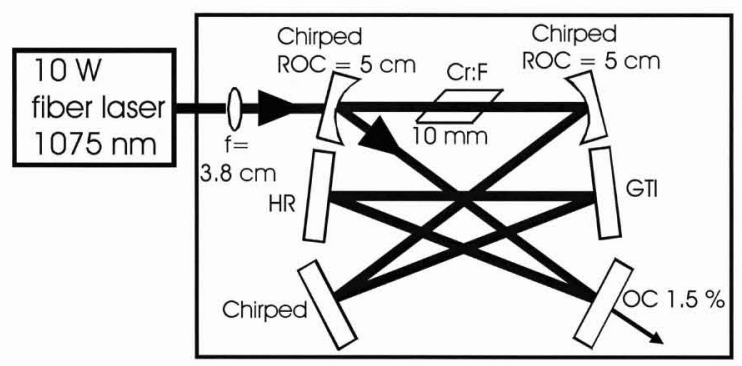

Fig. 1. The six-mirror ring-cavity setup. The mirror configuration shown yields the broadest mode-locked output spectrum. ROC, radius of curvature; HR, high reflector; OC, output coupler; GTI, Gires-Tournois interferometer. 
net cavity GDDs is accessible, resulting in different output spectra [Fig. 2]. A $1.5 \%$ output coupler and two concave chirped mirrors are used in all cavity configurations. A net dispersion of $260 \mathrm{fs}^{2}$, which produces the broadest spectrum, is achieved with a third chirped mirror, a Gires-Tournois interferometer mirror, and a low dispersion high reflector (Fig. 1). For this optimum cavity dispersion, the spectrum is peaked at $1280 \mathrm{~nm}$ with a 59-nm FWHM and pronounced wings ranging from 1080 to $1500 \mathrm{~nm}$. The inset of Fig. 2 shows the corresponding intensity autocorrelation after external compression using chirped mirrors. The pulse duration, assuming a hyperbolic secant pulse, is $30 \mathrm{fs}$, which deviates less than $10 \%$ from the transform limit. At $10 \mathrm{~W}$ of pump power the output power was $480 \mathrm{~mW}$ when mode locked and $580 \mathrm{~mW} \mathrm{cw}$. This is comparable with previous results at lower repetition rates.

The spectral width as a function of the net cavity GDD is shown in Fig. 3. From the measured spectral width we calculate the transform-limited pulse length, plotted as open circles in Fig. 3. A linear fit (solid line) of the pulse duration $\tau$ versus GDD gives a slope of $(0.055 \pm 0.007) \mathrm{fs}^{1}$. We compare these data with a theoretical model of solitonlike pulse formation ${ }^{11}$ that predicts that the transform-limited pulse duration will depend linearly on GDD. Assuming a nonlinear index of refraction ${ }^{2}$ of $n_{2}=(2 \pm 1) \times 10^{16} \mathrm{~cm}^{2} / \mathrm{W}$ and a beam waist in the crystal of $15 \mu \mathrm{m}$, we obtain a slope of $(0.070 \pm 0.035) \mathrm{fs}^{1}$. This result is shown as the dashed line in Fig. 3, obtained by adjusting the offset so that theory and data agree at GDD $=260 \mathrm{fs}^{2}$, where we measured the pulse duration. The agreement between theory and data is within the uncertainty and shows that the model of solitonlike pulse formation is applicable to this Cr:forsterite laser over at least this limited range of GDD. If the magnitude of the net intracavity GDD is decreased further, mode locking no longer occurs or is very unstable. We attribute this primarily to large oscillations in the GDD of the cavity mirrors. A related limitation of additional pulse shortening is the corresponding increase in peak power with smaller net GDD. Evidence for this is the tendency of double pulsing to occur for the cavity configuration with smallest GDD, which is eliminated by increasing the output coupling.

To further broaden the spectrum we sent the laser pulses through various HNLFs. ${ }^{12}$ These HNLFs are germanium-doped silica fibers with a small effective area $\left(\approx 14 \mu \mathrm{m}^{2}\right)$, nonlinear coefficient $\gamma=8.5 \mathrm{~W}^{1} \mathrm{~km}^{1}$, and zero dispersion near $1550 \mathrm{~nm}$ with low higher-order dispersion. We have experimented with individual $10-\mathrm{m}$ pieces of HNLF having slightly different core and cladding diameters, as well as hybrid fibers that were created by splicing together pieces of HNLF with different dispersions. The hybrid fiber was designed to be a dispersion-decreasing fiber at $1550 \mathrm{~nm}$. It consisted of four sections, each $1.5 \mathrm{~m}$ long, and the dispersion map at $1550 \mathrm{~nm}$ was $3.8,2.2, \quad 0.41$, and $2 \mathrm{ps} /(\mathrm{nm} \mathrm{km})$.

Even though these fibers were specifically designed for use near $1550 \mathrm{~nm}$, significant spectral broadening was obtained with the Cr:forsterite laser. Figure 4 summarizes the results of our fiber broadening experiments. For these results, the average power out of the laser was $480 \mathrm{~mW}$, and the center wavelength was $1280 \mathrm{~nm}$. We externally compressed the pulse to $30 \mathrm{fs}$ before coupling it into the nonlinear fibers. The average power obtained after the individual HNLF fibers was $260 \mathrm{~mW}$, while $180 \mathrm{~mW}$ was coupled through the hybrid fiber. Figure 4(a) shows the broadened spectra at the fiber outputs, measured with an optical spectrum analyzer. The spectra are clipped at the 1770-nm wavelength limit of the optical spectrum analyzer. Traces (i) and (ii) show the spectra after the HNLFs with calculated dispersion of 13.4 and $11.8 \mathrm{ps} /(\mathrm{nm} \mathrm{km})$, respectively, at $1280 \mathrm{~nm}$. Trace (iii) is the output of a 6-m hybrid fiber, consisting of four 1.5-m sections of HNLF having dispersion at $1280 \mathrm{~nm}$ of 10.8, 11.8, 13.4, and $14.4 \mathrm{ps} /(\mathrm{nm} \mathrm{km})$, respectively. For this hybrid fiber, light was launched into the end with dispersion of $10.8 \mathrm{ps} /(\mathrm{nm} \mathrm{km})$. For comparison, trace (iv) shows the spectrum after it has passed through $25 \mathrm{~km}$ of SMF-28 fiber, and trace (v) is the input spectrum.

To obtain information beyond $1770 \mathrm{~nm}$, we have also measured the spectra with a monochromator and

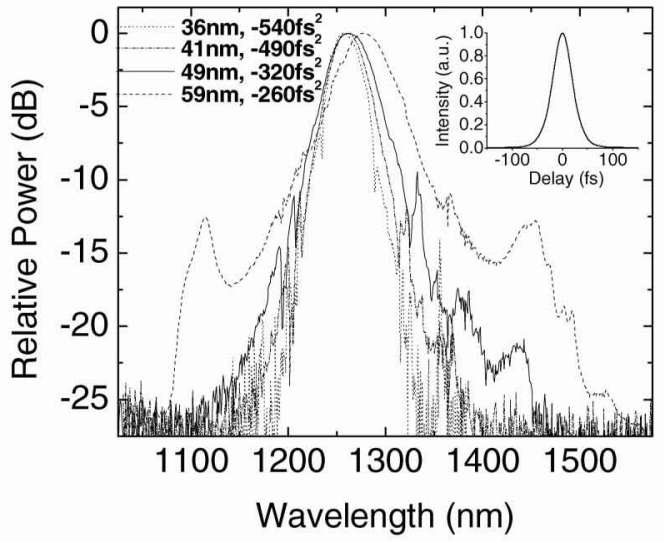

Fig. 2. Four mode-locked laser spectra for different values of the net cavity dispersion. Inset, autocorrelation trace for the cavity configuration yielding the largest FWHM spectrum.

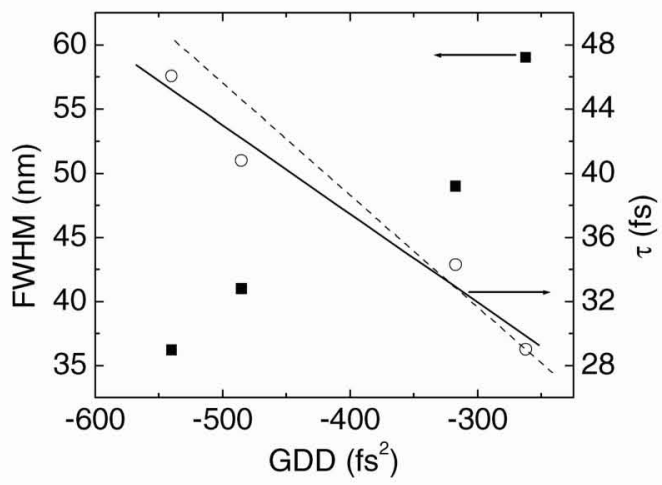

Fig. 3. Filled squares, measured FWHM of the laser spectra in Fig. 2; open circles, bandwidth-limited pulse duration calculated from the measured FWHM; solid line, linear fit to these values; dashed line, theoretical curve; see text. 
(a)
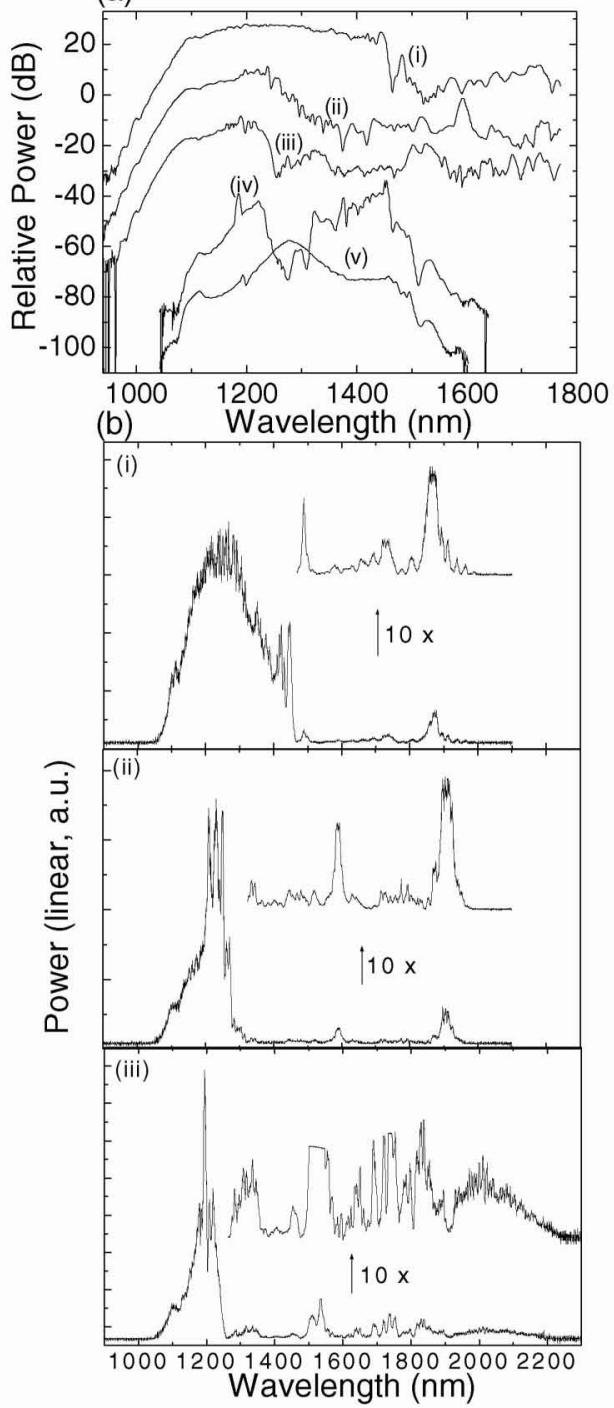

Fig. 4. Spectra from the output of various nonlinear fibers; see text. (a) Fiber output spectra recorded with an optical spectrum analyzer. (b) Spectra recorded with a monochromator to show the long-wavelength wings of the spectra.

an extended InGaAs photodiode suitable for detecting wavelengths as long as $2.2 \mu \mathrm{m}$. These results are shown in Fig. 4(b), where the spectral amplitudes are corrected to account for the wavelength-dependent monochromator and detector efficiencies. The three traces in this plot again show the spectra obtained with the two HNLFs and the hybrid fiber described above. As can be seen, the 6-m-long hybrid fiber yields an octave-spanning spectrum ranging from 1060 to near $2170 \mathrm{~nm}$. The octave, taken from 1075 to $2150 \mathrm{~nm}$, is reached at a level of $18 \mathrm{~dB}$ below the peak. The absolute spectral power at the edges of the octave is $110 \mu \mathrm{W} / \mathrm{nm}$ at $1075 \mathrm{~nm}$ and $45 \mu \mathrm{W} / \mathrm{nm}$ at $2150 \mathrm{~nm}$.

While the noise properties ${ }^{13,14}$ of this ultrabroad spectrum have not yet been fully investigated, we have evidence that a useful comb of coherent optical frequencies remains under the broad spectral envelopes of Fig. 4. A portion of the light near $1314 \mathrm{~nm}$ has been frequency doubled, and we have observed a heterodyne beat $(>30 \mathrm{~dB}$ signal-to-noise ratio in $400 \mathrm{kHz}$ bandwidth) between an individual mode of the frequency-doubled comb and the 657-nm stabilized diode laser of a calcium frequency standard. ${ }^{15}$ We have also observed a strong beat note between an individual mode of the stabilized comb and a cw laser operating near $1600 \mathrm{~nm}$, providing further evidence for the coherence of the comb.

The authors thank C. Chudoba, T. Clement, S. Gilbert, H. Kapteyn, J. Stone, B. Washburn, F. Wise, and V. Yakovlev for their valuable contributions. I. Thomann's e-mail address is isabell.thomann@ colorado.edu.

Note added in proof: We recently phase locked the broadened Cr:forsterite comb to optical and microwave standards and subsequently used the comb for measurements of optical frequency references at 1.3, 1.5, and $1.6 \mu \mathrm{m}$, thus demonstrating its utility for optical frequency metrology.

\section{References}

1. A. Seas, V. Petricevic, and R. R. Alfano, Opt. Lett. 17, 937 (1992).

2. V. Yanovsky, Y. Pang, F. Wise, and B. I. Minkov, Opt. Lett. 18, 1541 (1993).

3. Z. Zhang, K. Torizuka, T. Itatani, K. Kobayashi, T. Sugaya, T. Nakagawa, and H. Takahashi, Opt. Lett. 23, 1465 (1998).

4. C. Chudoba, J. G. Fujimoto, E. P. Ippen, H. A. Haus, U. Morgner, F. X. Kaertner, V. Scheuer, G. Angelow, and T. Tschudi, Opt. Lett. 26, 292 (2001).

5. B. E. Bouma, G. J. Tearney, I. P. Bilinsky, B. Golubovic, and J. G. Fujimoto, Opt. Lett. 21, 1839 (1996).

6. V. V. Yakovlev, V. Shcheslavskiy, and A. Ivanov, Appl. Phys. B 74, S145 (2002).

7. S. T. Cundiff, J. Ye, and J. L. Hall, Rev. Sci. Instrum. 72, 3749 (2001).

8. S. L. Gilbert, W. C. Swann, and T. Dennis, Proc. SPIE 4269, 184 (2001).

9. A. Bartels, T. Dekorsy, and H. Kurz, Opt. Lett. 24, 996 (1999).

10. I. Thomann, L. Hollberg, S. A. Diddams, and R. Equall, Appl. Opt. 42, 1661 (2003).

11. F. Krausz, M. E. Fermann, T. Brabec, P. F. Curley, M. Hofer, M. H. Ober, C. Spielmann, E. Winter, and A. J. Schmidt, IEEE J. Quantum Electron. 28, 2097 (1992).

12. J. W. Nicholson, M. F. Yan, P. Wisk, J. Fleming, F. DiMarcello, E. Monberg, A. Yablon, C. G. Jørgensen, and T. Veng, Opt. Lett. 28, 643 (2003).

13. K. L. Corwin, N. R. Newbury, J. M. Dudley, S. Coen, S. A. Diddams, K. Weber, and R. S. Windeler, Phys. Rev. Lett. 90, 113904 (2003).

14. N. R. Newbury, B. R. Washburn, K. L. Corwin, and R. S. Windeler, Opt. Lett. 28, 944 (2003).

15. C. W. Oates, F. Bondu, R. W. Fox, and L. Hollberg, Eur. Phys. J. D 7, 449 (1999). 